\title{
MOLECULAR OBSERVATIONS OF SHOCKS AND OUTFLOWS
}

\author{
R. BACHILLER \\ Observatorio Astronómico Nacional \\ Apartado 1143, E-28800 Alcalá de Henares, Spain
}

\begin{abstract}
We discuss recent high-sensitivity observations carried out with the IRAM instruments of the shock chemistry associated with bipolar outflows. We highlight the recent mm-wave studies of L 1157, a highlycollimated outflow driven by a Class 0 source. These observations illustrate nicely the chemical segregation produced by the outflow. Different molecular lines are observed to trace different components of the gas. Some molecules are abundant in the quiescent medium but are not observed in the shock (e.g. $\mathrm{C}_{3} \mathrm{H}_{2}, \mathrm{~N}_{2} \mathrm{H}^{+}, \mathrm{H}^{13} \mathrm{CO}^{+}, \mathrm{DCO}^{+}$), whereas some other molecules are very enhanced at the shocked region (e.g. $\mathrm{SiO}, \mathrm{CH}_{3} \mathrm{OH}, \mathrm{H}_{2} \mathrm{CO}, \mathrm{HCN}$, $\mathrm{CN}$, SO, $\mathrm{SO}_{2}, \mathrm{H}_{2} \mathrm{CS}$ ). We briefly discuss the chemistry of the most important molecules, devoting special attention to the species which are thought to be abundant in interstellar ice mantles.
\end{abstract}

\section{Introduction}

Young stars and protostars produce powerful bipolar outflows which are observable over a wide range of wavelengths, from the ultraviolet to the radio. In many cases, the bipolar outflow is the first clear sign that reveals the formation of a new star. Bipolar outflows are observed even around the youngest protostars already identified (the so-called "Class 0" objects). In fact, outflows from "Class 0" sources are particularly violent: the mechanical power can reach $30 \%$ of the bolometric luminosity of the central engine instead of the $0.001-0.01 \%$ observed in most standard outflows. The presence of vigorous outflow activity in such young objects underscores the importance of the bipolar outflows in the formation of stars. Outflows seem to be essential in star formation. In particular, outflows can carry away the excess of angular momentum from the contracting molecular cloud, and can limit the mass and size of the protostellar infalling condensation. 
Such violent outflows accompanying the birth of a new star impact on the remaining molecular cloud dispersing the surrounding molecular material, and determine the evolution of the dense core where the star was born. Outflows drive shock waves that compress and heat the gas triggering important chemical processes that do not operate in quiescent environments. The particularly violent outflows from Class 0 protostars are expected to produce dramatic effects on the structure and on the chemical composition of the surrounding molecular cloud. In addition, outflows from Class 0 sources are highly collimated (Bachiller 1996), so the region where the shock occurs is well defined spatially, and well separated from the vicinity of the driving source which retains the properties of the quiescent ambient gas. Processes such as endothermic reactions, sublimation of ices, and disruption of dust grain cores lead to a "shock-chemistry" which is radically different from that operating in the quiescent medium. Some of these processes are fast, and the cooling times of the shocked region are short, making the chemistry strongly time-dependent.

Recent observations with high angular resolution and sensitivity are providing important insights in these transient phenomena. We discuss here one of the most illustrative cases of outflows from Class 0 sources: the bipolar molecular outflow in the dark cloud L 1157. Due to its proximity $(440 \mathrm{pc})$, high collimation, favorable orientation in the sky, and high column densities in the shocked region, this is one of the best objects to study the rich phenomenology associated with young bipolar outflows.

\section{L 1157-mm as an example of a Class 0 source}

The highly collimated molecular outflow in L 1157 was discovered by Umemoto et al. (1992). The outflow is driven by L 1157-mm (IRAS 20386+6751), a very cold source of about $11 \mathrm{~L}_{\odot}$, embedded within a dense $\mathrm{NH}_{3}$ core (Bachiller et al. 1993). The central source presents all the attributes of Class 0 sources, including a relatively strong flux density at millimeter wavelengths due to thermal dust emission. Such a high flux density makes it possible to obtain detailed images of the protostellar vicinity with mm-wave interferometers (e.g. Gueth et al. 1996). A recent high-angular resolution study by Guilloteau et al. (1996) shows that the continuum emission near $\lambda 2.7 \mathrm{~mm}$ consists of a $35 \mathrm{mJy}$ source of size $\leq 1^{\prime \prime}$, surrounded by spatiallyextended low-level emission. The extended emission seems to arise from the heated edges of the cavity excavated by the high-velocity bipolar outflow. High-angular resolution images of the source in the $J=1-0$ line of $\mathrm{CO}$, ${ }^{13} \mathrm{CO}$, and $\mathrm{C}^{18} \mathrm{O}$ lead to a picture in which the $\mathrm{C}^{18} \mathrm{O}$ emission is associated with the protostellar condensation, whereas the ${ }^{13} \mathrm{CO}$ line emission mostly originates from a more extended envelope and the limb-brightened edges 


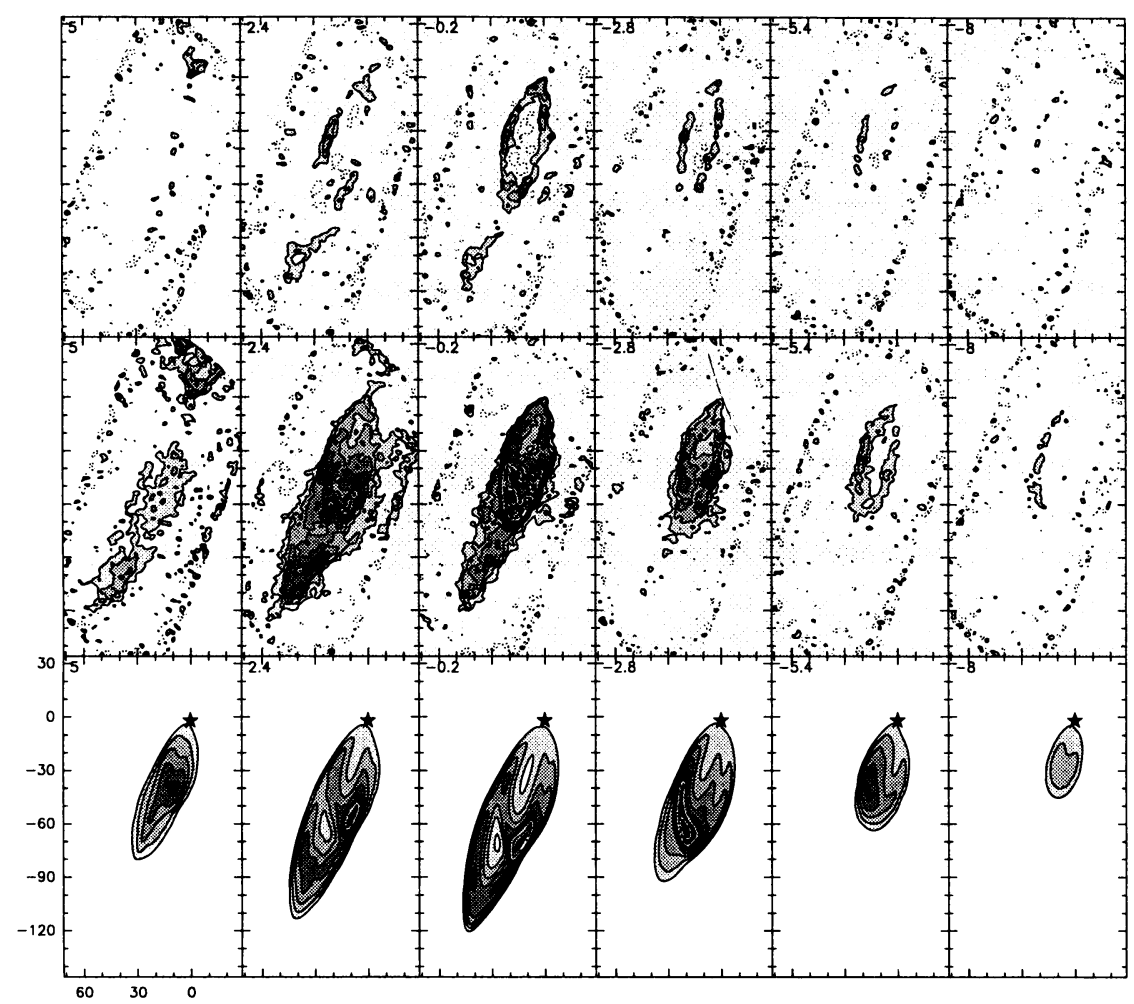

Figure 1. CO 1-0 emission from the blueshifted lobe of the L 1157 outflow integrated over velocity intervals of $2.6 \mathrm{~km} / \mathrm{s}$. Position offsets are in " with respect to L 1157-mm, the outflow exciting source. Top row.- maps obtained from purely interferometric IRAM data. Middle row.- maps obtained after inclusion of short spacing information obtained at the IRAM 30-m telescope. Bottom row.- synthetic maps obtained with a precessing episodic jet model smoothed to the resolution of the observations (from Gueth et al. 1996).

of the outflow. The CO line wings trace the bulk of the outflowing material. Possible gravitational infall motions are recognized by comparing the self-reversed ${ }^{13} \mathrm{CO}$ profiles with the narrow $\mathrm{C}^{18} \mathrm{O}$ lines.

\section{The CO bipolar outflow in $\mathrm{L} \mathbf{1 1 5 7}$}

The bipolar outflow in L 1157 has a size of about $0.6 \mathrm{pc}$, and it is highly collimated but asymmetric (Umemoto et al. 1992). The southern blue-shifted lobe is more compact and brighter than the northern lobe, suggesting that the blue-shifted gas is shocking against some kind of obstacle, whereas the northern lobe is flowing more freely. The brightness of the blue-shifted lobe makes it one of the best places to study the structure of the shock. Fig- 
ure 1 from Gueth et al. (1996) shows velocity channel CO images of the blue-shifted lobe. The images reveal at least two prominent limb-brightened cavities which seem to be created by the propagation of large bow shocks. These observations also illustrate the importance of combining single-dish data with the interferometric data. In fact, with only the purely interferometric images (top row of the figure), one could think that the cavities are empty structures. When one adds the zero-spacing information (middle row) it is shown that there is significant $\mathrm{CO}$ emission arising from the inner part of the cavities. Interestingly, the two cavities are not well aligned on a single line passing through the exciting source, L $1157-\mathrm{mm}$, as if the axis of the underlying jet had precessed from the first ejection event to the second one. A simple spatio-kinematic model in which the jet precesses on a narrow cone (of opening angle close to $6^{\circ}$ ) provides an accurate description of the observations (bottom row of the figure).

Indeed the sharp increase of the $\mathrm{CO}$ brightness temperature towards the blue-shifted lobe in L 1157 could be due to the gas heating produced by the shock. Multiline observations of ammonia, the best interstellar thermometer, confirm that this is the case, and provide a good estimate of the gas kinetic temperature in the shock, which results to be in the range $60-100 \mathrm{~K}$ (Bachiller et al. 1993). The bow shocks at the head of the cavities are particularly well observed in $\mathrm{NH}_{3}(3,3)$ emission, and the VLA images shown in Fig. 2 (from Tafalla \& Bachiller 1995, 1996) reveal the hot regions of the bow shock structures seen in the high-velocity CO. The ammonia abundance can be estimated in both the quiescent gas and in the high-velocity outflow, and it results that this abundance is enhanced by at least a factor of 10 in the shocked region.

\section{Chemistry in the $\mathrm{L} \mathbf{1 1 5 7}$ outflow}

In addition to ammonia, some other molecular abundances have long been suspected to be enhanced as a result of the action of bipolar outflows on the surrounding gas. One of the most extreme examples is $\mathrm{SiO}$, whose gas phase abundance is known to be enhanced by several orders of magnitude at the heads and along the axes of some molecular outflows (Bachiller et al. 1991; Martín-Pintado et al. 1992; McMullin et al. 1994). The case of L 1157 is not less spectacular, and a wealth of observations (Mikami et al. 1992, Zhang et al. 1996, Avery \& Chiao 1996) indicate that $\mathrm{SiO}$ is enhanced by a factor of $\sim 10^{6}$ in the shocked region.

Spectral line surveys at $\mathrm{mm}$ wavelengths are recognized to be the best tool to explore the chemical composition of the vicinity of low-mass YSOs. Recent studies have considered two important Class 0 sources : NGC1333 IRAS4 (Blake et al. 1995) and IRAS16293 (Blake et al. 1994; van Dishoeck 


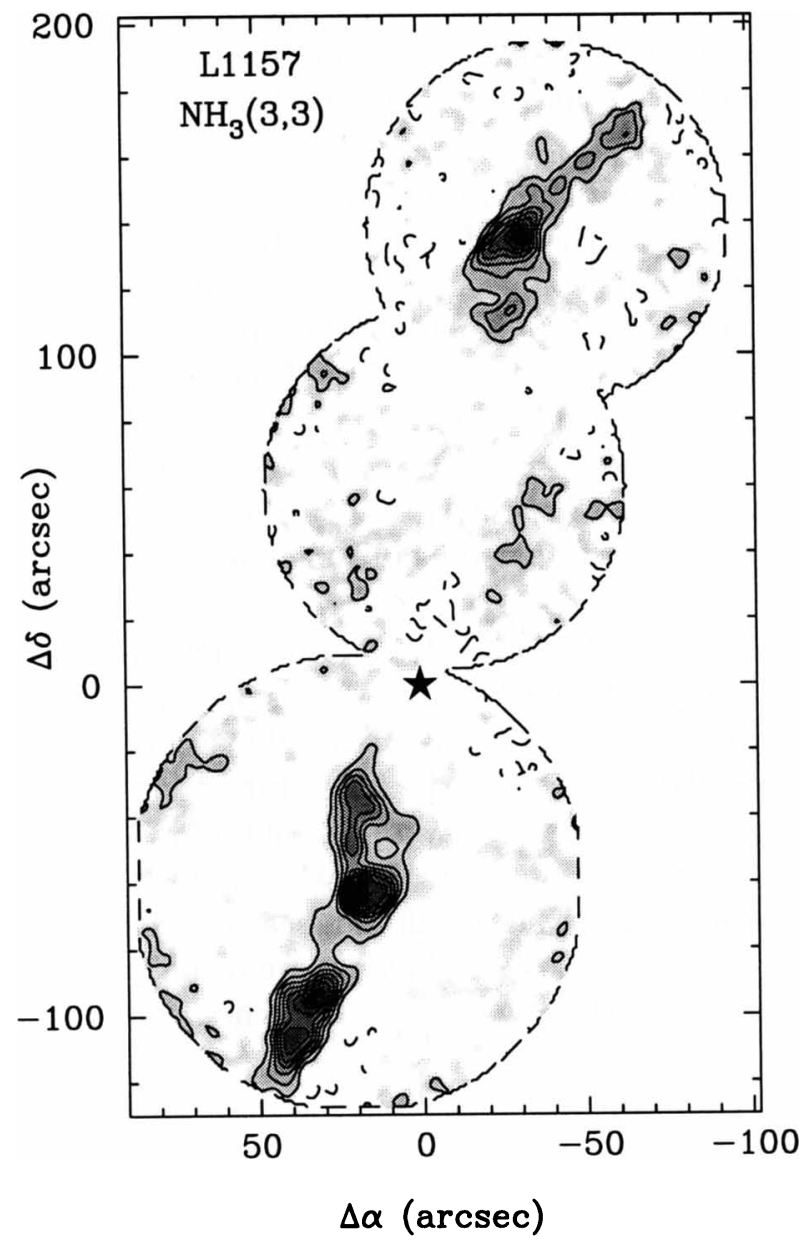

Figure 2. Map of integrated intensity of the $\mathrm{NH}_{3}(3,3)$ line in $\mathrm{L} 1157$ obtained with the VLA (D configuration, $5^{\prime \prime}$ resolution). The map center is on the exciting source L 1157-mm. The southern lobe consists of blue-shifted emission (see CO images in Fig. 1), whereas the red-shifted emission is in the northern lobe (from Tafalla \& Bachiller $1995,1996)$.

et al. 1995). Very recently, Bachiller \& Pérez (1996) have carried out a survey of the blue-shifted lobe in L 1157 to study the chemical composition of the gas affected by the passage of the shock. We observed about 50 different lines of 27 molecules (including rare isotopic species) toward the position of the central source $(0,0)$ and several positions at the shocked regions, in particular the two prominent $\mathrm{NH}_{3}$ peaks of the blue-shifted 
lobe: $\mathrm{B} 1$ at position $\left(20^{\prime \prime},-60^{\prime \prime}\right)$, and $\mathrm{B} 2$ at $\left(35^{\prime \prime},-95^{\prime \prime}\right)$. Some of the profiles are shown in Fig. 3. Indeed, the narrow line profiles observed toward the position of the source arise from cold quiescent gas, whereas toward the bow shock region the profiles are dominated by the broad lines associated with the shock.

One of the first results emerging from these observations is that some molecular lines such as those of $\mathrm{C}_{3} \mathrm{H}_{2}, \mathrm{~N}_{2} \mathrm{H}^{+}, \mathrm{H}^{13} \mathrm{CO}^{+}$, and $\mathrm{DCO}^{+}$are only observed toward the cold gas condensation around the exciting source, whereas some other molecules (such as $\mathrm{SiO}$ ) only trace the warm gas in the shock. This result is important for studies of the structure of the central infalling condensation, since one can avoid the outflow contribution to the line profiles by observing the source vicinity in lines of $\mathrm{C}_{3} \mathrm{H}_{2}, \mathrm{~N}_{2} \mathrm{H}^{+}, \mathrm{H}^{13} \mathrm{CO}^{+}$, and $\mathrm{DCO}^{+}$. This does not mean that the outflowing gas is fully free of these molecules, but the column densities are insufficient to produce significant wings in the profiles.

All the other detected molecules $\left(\mathrm{SiO}, \mathrm{CH}_{3} \mathrm{OH}, \mathrm{H}_{2} \mathrm{CO}, \mathrm{HCO}^{+}, \mathrm{HCN}\right.$, $\mathrm{HNC}, \mathrm{CN}, \mathrm{SO}, \mathrm{SO}_{2}, \mathrm{CS}, \mathrm{OCS}, \mathrm{H}_{2} \mathrm{CS}$ ) present emission in the outflow. An interesting effect concerns the relative intensity of the lines at the two observed positions B1 and B2, in the blue-shifted lobe. Both positions are almost equally strong in the $\mathrm{CO}$ isotopes, $\mathrm{SiO}$ and $\mathrm{CH}_{3} \mathrm{OH}$. However, the peak $\mathrm{B} 1$ is more prominent in $\mathrm{H}_{2} \mathrm{CO}, \mathrm{HCO}^{+}$, and $\mathrm{CN}$, whereas the $\mathrm{B} 2$ peak is much more prominent in most sulphur-bearing molecules like $\mathrm{SO}, \mathrm{SO}_{2}$, and OCS. This cannot be explained as a mere result of possible differences in the excitation. Important chemical differences seem to exist between positions $\mathrm{B} 1$ and $\mathrm{B} 2$.

The abundances of the different molecules have been estimated from the ratios of the column densities to the $\mathrm{CO}$ column density, and by assuming a standard $\mathrm{CO} / \mathrm{H}_{2}$ ratio. The $\mathrm{CO}$ column densities in the quiescent gas and in the outflow have been derived from the $\mathrm{C}^{18} \mathrm{O}$ lines and the $\mathrm{CO}$ line wings, respectively, by assuming optically thin emission and thermalization. For the other molecules we used a Large-Velocity-Gradient (LVG) code, or we did similar assumptions as in the case of CO. The enhancement factors of the different species in the shock are thus estimated as the ratios of the abundances estimated in the shock to those estimated in the quiescent gas. I next discuss some particular molecules, devoting special attention to the species that are thought to be abundant in the dust grain mantles, since such molecules are expected to be desorbed from grains by the action of the shocks.

Tielens \& Whittet (this volume) give an updated list of molecular species detected in the solid phase in star-forming regions. Such molecules include $\mathrm{H}_{2} \mathrm{O}, \mathrm{CH}_{3} \mathrm{OH}, \mathrm{CO}, \mathrm{CO}_{2}, \mathrm{CH}_{4}$, and $\mathrm{OCS}$. Methanol $\left(\mathrm{CH}_{3} \mathrm{OH}\right)$, with an abundance of a few percent, is thought to be one of the most abun- 


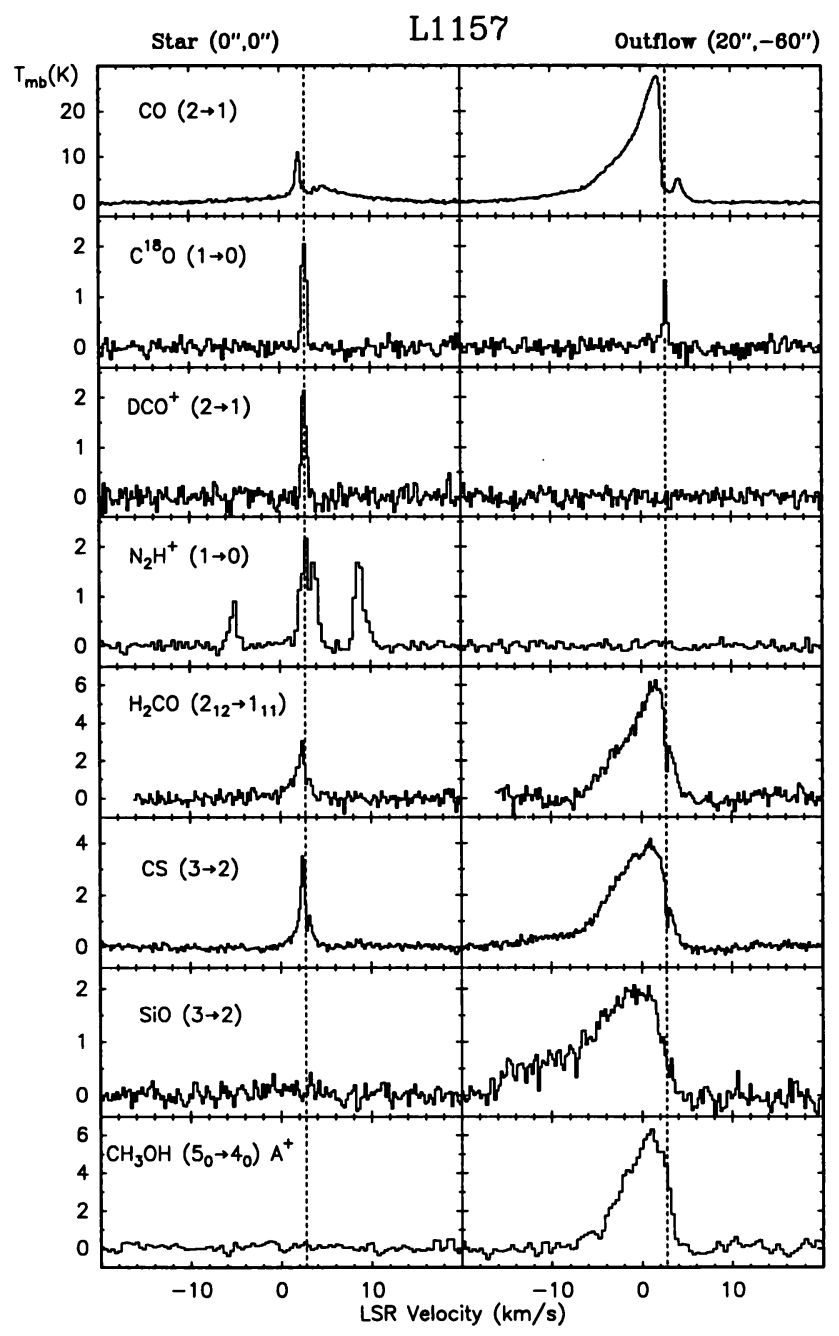

Figure 3. Molecular line profiles observed towards two representative positions in the L 1157 outflow: the $(0,0)$ position on the central star and position $\left(20^{\prime \prime},-60^{\prime \prime}\right)$ on one of the bow shocks in the blue-shifted lobe. The lines toward the star positions are narrow, since they arise from quiescent material associated with the dense core. The lines observed toward the outflow are very broad and blue-shifted with respect to the ambient gas velocity (marked with a dashed line). 
dant molecule in ices, after water (Allamandola et al. 1992). Indeed, the millimeter-wave observations of L 1157 (Bachiller et al. 1995) show that the abundance of methanol is enhanced by a factor of 400 in the shock (see also Avery \& Chiao 1996). Such methanol enhancements have been observed in some other outflows (Sandell et al. 1994 ; Bachiller et al. 1995 ; Garay et al. 1996).

There is increasing evidence that high abundances of methanol in the gas phase are able to drive a chemistry completely different from the usual chemistry operating in dark clouds. In particular, laboratory experiments show that a significant fraction of the methanol released to the gas phase could produce $\mathrm{H}_{2} \mathrm{CO}$ in a relatively short time (e.g., Bernstein et al. 1995). Solid formaldehyde $\left(\mathrm{H}_{2} \mathrm{CO}\right)$ has also been recently discovered in the vicinity of protostars (Schutte et al. 1996). Our observations of L 1157 indicate that $\mathrm{H}_{2} \mathrm{CO}$ is enhanced by a factor close to 100 in the shocked region. The chemistry of formaldehyde is probably closely related to that of methanol in both solid and gas phases.

The chemistry of sulfur is also severely affected by shocks (e.g. Pineau de Forêts et al. 1993), and SO has been found to be enhanced in several molecular outflows (Martín-Pintado et al. 1992; Schmid-Burgk \& Muders 1995; Chernin et al. 1994). The observations of L 1157 reveal significant enhancements of $\mathrm{SO}, \mathrm{SO}_{2}$, OCS, and $\mathrm{H}_{2} \mathrm{CS}$ in the shocked region, with the peculiarity, as mentioned above, that the $\mathrm{B} 2$ peak is richer in most sulfurbearing molecules. It is interesting to note that $\mathrm{H}_{2} \mathrm{~S}$ was only tentatively detected towards $\mathrm{B} 1 . \mathrm{H}_{2} \mathrm{~S}$ is probably formed by grain-surface reactions (Duley et al. 1980) and could be directly released from the mantles. $\mathrm{H}_{2} \mathrm{~S}$ is then expected to produce $\mathrm{SO}$ and $\mathrm{SO}_{2}$ very quickly (in a few $10^{3} \mathrm{yr}$ ) via gas-phase reactions with $\mathrm{H}, \mathrm{OH}$, and $\mathrm{O}_{2}$ (Millar 1993, and this volume). Thus, the differences in the $\mathrm{SO}, \mathrm{SO}_{2}$, and $\mathrm{H}_{2} \mathrm{~S}$ abundances between $\mathrm{B} 1$ and $\mathrm{B} 2$ could be related to their differences of age. It is indeed expected that $\mathrm{B} 2$ is older than $\mathrm{B} 1$, since $\mathrm{B} 2$ is more distant to the central star.

Molecular spectroscopy is also a powerful tool to investigate the physical conditions in the shocked gas. As discussed above, $\mathrm{NH}_{3}$ provides good estimates of the kinetic temperature. Once the temperature is known, multiline studies of $\mathrm{SiO}$ and $\mathrm{CH}_{3} \mathrm{OH}$ provide reliable estimates of the volume densities. In the case of the L 1157 bow shock, the density is found to be in excess of $10^{6} \mathrm{~cm}^{-3}$ (Bachiller et al. 1993, 1995). The region emitting the radio lines is significantly colder $\left(\mathrm{T}_{K} \sim 10^{2} \mathrm{~K}\right)$ than the gas traced by the near infrared lines of $\mathrm{H}_{2}$ (in which $\mathrm{T}_{K} \sim$ a few $10^{3} \mathrm{~K}$ ). However, the images of the $\mathrm{L} 1157$ outflow in the $\mathrm{H}_{2}$ lines near $2.1 \mu \mathrm{m}$ (Davis \& Eisloeffel 1995) also reveal the bow shock structures similar to those seen in $\mathrm{CO}$ and $\mathrm{NH}_{3}$. It seems that sharp gradients in the kinetic temperature are present across the bow shocks. 


\section{References}

Allamandola L.J., Sandford S.A., Tielens A.G.G.M., Herbst T. 1992, ApJ 399, 134

Avery, L. W., Chiao, M. 1996, ApJ 463, 642

Bachiller, R. 1996, ARAA 34, 111

Bachiller, R., Liechti, S., Walmsley, C.M., Colomer, F. 1995, A\&A 295, L51

Bachiller, R., Martín-Pintado, J., Fuente, A. 1991, A\&A 243, L21

Bachiller, R., Martín-Pintado, J., Fuente, A. 1993, ApJ 417, L45

Bachiller, R., Pérez, M. 1996, in preparation

Bernstein, M.P., Sandford, S.A., Allamandola, L.J., Chang, S., Scharberg, M.A. 1995, ApJ 454, 327

Blake, G.A., van Dishoeck, E.F., Jansen, D.J., Groesbeck, T.D., Mundy, L.G. 1994, ApJ 428,680

Blake, G.A., Sandell, G., van Dishoeck E.F., Groesbeck, T.D., Mundy, L.G., Aspin, C. 1995, ApJ 441, 689

Chernin, L.M., Masson, C.R., Fuller, G. 1994, ApJ 436, 741

Davis, J.J., Eisloeffel, J. 1995, A\&A 300, 851

Duley, W.W., Millar, T.J., Williams, D.A. 1980, MNRAS 192, 945

Garay, G., Koehnenkamp, I., Rodríguez, L.F. 1996, The ESO Messenger 84, 31

Gueth, F., Guilloteau, S., Bachiller, R. 1996, A\&A 307, 891

Guilloteau, S., Gueth, F., Bachiller, R. 1996, submitted to A\&A

Martín-Pintado, J., Bachiller, R., Fuente, A. 1992, A\&A 254, 315

McMullin, J.P., Mundy, L.G., Blake, G.A. 1994, ApJ 437, 305

Millar, T.J. 1993, in Dust and Chemistry in Astronomy, eds. T.J. Millar \& D.A. Williams (Bristol, IOP publishing)

Mikami, H., Tomofumi, U., Yamamoto, S., Saito, S. 1992, ApJ 392, L87

Pineau des Forêts, G., Roueff, E., Schilke, P., Flower, D. 1993, MNRAS 262, 915

Sandell, G., Knee, L.B.G., Aspin, C., Robson, I.E., Russell, A.P.G. 1994, A\&A 285, L1

Schmid-Burgk, J., Muders, D. 1995, in Stellar and Circumstellar Astrophysics, eds. G. Wallerstein, A. Noriega-Crespo (ASP Conf. Ser., San Francisco)

Schutte, W.A., Gerakines, P.A., Geballe, T.R., van Dishoeck, E.F., Greenberg, J.M. 1996, A\&A 309, 633

Tafalla, M., Bachiller, R. 1995, ApJ 443, L37

Tafalla, M., Bachiller, R. 1996, in preparation

Umemoto, T., Iwata, T., Fukui, Y., Mikami, H., Yamamoto, S., Kamaya, O., Hirano, N. 1992, ApJ 392, L83

van Dishoeck, E.F., Blake, G.A., Jansen, D.J., Groesbeck, T.D. 1995, ApJ 447, 760

Zhang, Q., Ho, P.T.P., Wright, M.C.H., Wilner, D.J. 1995, ApJ 451, L71 


\section{Discussion}

Wootten: This morning, Tielens gleefully stashed nitrogen in $\mathrm{N}_{2}$ on grain mantles, where it remains unobservable. Although you find $\mathrm{HCO}^{+}$in flows, you do not observe $\mathrm{N}_{2} \mathrm{H}^{+}$, produced by the same $\mathrm{H}_{3}^{+}$chemistry. You do find enhanced $\mathrm{NH}_{3}$, presumably from grain mantles. Either a bizarre chemistry operates (perhaps you should include NO in your survey) or $\mathrm{N}_{2}$, though a stable molecule, does not survive mantle escape. There seems a problem with nitrogen.

Bachiller: In my opinion, it is not well known what are the N-bearing species present in grain mantles. On the other hand, the shock-chemistry of nitrogen is poorly understood. In addition, $\mathrm{N}_{2} \mathrm{H}^{+}$is peculiar even in 'standard' molecular clouds (sometimes $\mathrm{N}_{2} \mathrm{H}^{+}$peaks offset from most molecules). More observational work is clearly necessary. Your suggestion of observing NO in L1157 is a very good one.

Ohishi: You mention that $\mathrm{HCO}^{+}$is enhanced in $\mathrm{L} 1157$ but $\mathrm{DCO}^{+}$is not. Why is this?

Bachiller: The $\mathrm{HCO}^{+}$enhancement is difficult to quantify due to the high opacities of the ambient lines, which make the abundance in the quiescent phase very uncertain. If $\mathrm{HCO}^{+}$were only moderately enhanced, the wings could remain undetected in $\mathrm{H}^{13} \mathrm{CO}^{+}$and $\mathrm{DCO}^{+}$due to the sensitivity limitations, although the isotopic abundance ratios could be standard. Anyway, the $\mathrm{H}^{13} \mathrm{CO}^{+}$and $\mathrm{DCO}^{+}$lines are very good to study the central source because they are narrow (no detectable outflow contamination), relatively strong, and have a high intensity contrast in the source vicinity.

Mauersberger: How can one explain multiple or perpendicular outflows?

Bachiller: Most (if not all) of the known multiple and perpendicular outflows have binary stars or stellar clusters at their origin. Precession in single outflows is also observed, but usually precession seems to occur over relatively small angles.

Val'tts: Are there methanol masers at $44 \mathrm{GHz}$ in this outflow?

Bachiller: We carried out a quick search for $44 \mathrm{GHz}$ mehanol masers in L1157, with the Yebes radiotelescope, without success. More careful searches would be worthwhile.

Takano: Your $\mathrm{NH}_{3}$ data in $\mathrm{L} 1157$ show clear enhancement in intensity for $\mathrm{NH}_{3}$ $(3,3)$, but not for $\mathrm{NH}_{3}(2,2)$ in the shock region. Can you interpret this result by a high ortho/para ratio of $\mathrm{NH}_{3}$ ? $\mathrm{NH}_{3}(3,3)$ belongs to the ortho species. If $\mathrm{NH}_{3}$ is produced on cold grains and evaporated, the ortho/para ratio can be high. What was the actual ortho/para ratio?

Bachiller: Our $\mathrm{NH}_{3}$ observations (Bachiller et al. 1993, ApJ 417, L45) also include the $(4,4)$ line. The corresponding Boltzmann plots are consistent with an ortho/para ratio of $\sim 2$, and the values derived for the kinetic temperatures are fully consistent with the measured intensities of the $(4,4)$ line. 